\title{
Resonant-Plasmon-Assisted Subwavelength Ablation by a Femtosecond Oscillator
}

\author{
Liping Shi (石理平), ${ }^{1,2, *}$ Bianca Iwan, ${ }^{1,4}$ Quentin Ripault, ${ }^{3}$ José R. C. Andrade, ${ }^{1,2}$ Seunghwoi Han, ${ }^{5}$ \\ Hyunwoong Kim, ${ }^{5}$ Willem Boutu, ${ }^{3}$ Dominik Franz, ${ }^{3}$ Rana Nicolas, ${ }^{3}$ Torsten Heidenblut, ${ }^{6}$ \\ Carsten Reinhardt, ${ }^{7,11}$ Bert Bastiaens, ${ }^{8}$ Tamas Nagy, ${ }^{1,9,10}$ Ihar Babuskin, ${ }^{1,10}$ Uwe Morgner, ${ }^{1,2}$ Seung-Woo Kim, ${ }^{5}$ \\ Günter Steinmeyer, ${ }^{10}$ Hamed Merdji, ${ }^{3}$ and Milutin Kovačev ${ }^{1,2, \uparrow}$ \\ ${ }^{1}$ Institut für Quantenoptik, Leibniz Universität Hannover, Welfengarten 1, 30167 Hannover, Germany \\ ${ }^{2}$ QUEST, Centre for Quantum Engineering and Space-Time Research, 30167 Hannover, Germany \\ ${ }^{3}$ LIDYL, CEA, CNRS, Université Paris-Saclay, CEA Saclay, 91191 Gif-sur-Yvette, France \\ ${ }^{4}$ Stanford PULSE Institute, SLAC National Accelerator Laboratory, \\ 2575 Sand Hill Rd, Menlo Park 94025, California, USA \\ ${ }^{5}$ Department of Mechanical Engineering, Korea Advanced Institute of Science and Technology (KAIST), \\ Science Town, Daejeon 305-701, South Korea \\ ${ }^{6}$ Institut für Werkstoffkunde, Leibniz Universität Hannover, \\ An der Universität 2, 30823 Garbsen, Hannover, Germany \\ ${ }^{7}$ Laser Zentrum Hannover e.V., Hollerithallee 8, 30419 Hannover, Germany \\ ${ }^{8}$ Laser Physics and Nonlinear Optics, MESA+Institute for Nanotechnology, \\ University of Twente, Enschede, Netherlands \\ ${ }^{9}$ Laser-Laboratorium Gottingen e.V., Hans-Adolf-Krebs-Weg 1, 37077 Göttingen, Germany \\ ${ }^{10}$ Max-Born-Institut, Max-Born-Straße 2a, 12489 Berlin, Germany \\ ${ }^{11}$ Hochschule Bremen City University of Applied Sciences, Neustadtswall 30, 28199 Bremen, Germany
}

(Received 13 February 2017; revised manuscript received 8 December 2017; published 2 February 2018)

\begin{abstract}
We experimentally demonstrate the use of subwavelength optical nanoantennas to assist a direct nanoscale ablation using the ultralow fluence of a Ti:sapphire oscillator through the excitation of surface plasmon waves. The mechanism is attributed to nonthermal transient unbonding and electrostatic ablation, which is triggered by the surface plasmon-enhanced field electron emission and acceleration in vacuum. We show that the electron-driven ablation appears for both nanoscale metallic as well as dielectric materials. While the observed surface plasmon-enhanced local ablation may limit the applications of nanostructured surfaces in extreme nonlinear nanophotonics, it, nevertheless, also provides a method for nanomachining, manipulation, and modification of nanoscale materials. Collateral thermal damage to the antenna structure can be suitably avoided, and nonlinear conversion processes can be stabilized by a dielectric overcoating of the antenna.
\end{abstract}

DOI: 10.1103/PhysRevApplied.9.024001

\section{INTRODUCTION}

Pulsed laser ablation is defined as the removal of material from a target by the direct absorption of photon energy and is of great interest in scientific research and industrial applications, such as laser micromachining, deposition of thin films, and the creation of new materials [1,2]. The high ablation threshold of many solids typically requires the utilization of high laser fluences operating at a low repetition rate. However, high fluences may lead to various undesired results, such as collateral thermal damage, which may appear around the laser spot [3-5]. It has been demonstrated that the

\footnotetext{
*Corresponding author. shi@iqo.uni-hannover.de

Corresponding author. kovacev@iqo.uni-hannover.de
}

threshold fluence decreases with pulse duration, therefore, compared to nanosecond lasers, the ablation process can be initiated by a femtosecond laser with much lower pulse energies. Nevertheless, this rather low pulse energy is capable of fabricating more precise features on solid surfaces with a reduced heat-affected area surrounding the ablated region [6,7]. This reduction is due to the fact that the pulse width of the femtosecond laser is much shorter than the electron-phonon coupling time (several picoseconds). Consequently, the localized energy deposition is rapidly removed from the region of ablation. It is widely believed that nanoscale small material manipulation will have a big impact in the near future [8]. However, due to the optical diffraction limit, it is difficult to achieve nanometric resolution by direct femtosecond laser ablation [9].

In order to overcome this apparent limitation, tipenhanced near-field material ablation has been explored 
by several groups [10-13]. This ablation method is based on the combination of a pulsed laser and a nanometric sharp tip, usually from an atomic force microscope, which is leveled at a distance of a few nanometers above the sample surface. Under illumination with light, the metallic tip amplifies the radiation intensity around the apex. Subwavelength craters result from the nanoscale heating, and subsequent phase transitions may appear on the sample surface.

In this article, we demonstrate the utilization of resonant plasmonic nanoantennas [14] to achieve subwavelength material ablation. The collective oscillation of free electrons within the skin layer of a nanoantenna may produce high electric fields within an extremely small area, which then results in an order-of-magnitude enhancement of the local field strength in the vicinity of a sharp feature or in the feed gap of a dimeric nanoantenna [15]. This concept of local nearfield enhancement has been exploited, such as for near-field scanning optical microscopy [16], photodetectors [17], photovoltaic cells [18], thermal emitters [19], saturable absorbers [20], extreme-ultraviolet emission [21], single-molecule fluorescence enhancement [22], harmonic generation enhancement [23,24], and optical tweezers [25]. Compared to metallic nanotips, precisely fabricated resonant nanoantennas enable us to tailor the electric near fields with a higher level of control and also offer a more substantial enhancement of incident radiation intensity. Therefore, the resonant plasmonic nanoantenna provides a platform to assist nanoscale material ablation directly by a femtosecond oscillator operating at ultralow fluence and a high repetition rate.

Usually, collateral thermal damage becomes more obvious when the area of interest reaches the nanoscale, as imposed by the larger surface-to-volume ratio and the smaller heat capacity of nanomaterials. For instance, the thermal damage threshold of a gold $(\mathrm{Au})$ nanoparticle with a diameter of $100 \mathrm{~nm}$ is less than $0.01 \mathrm{~J} / \mathrm{cm}^{2}$ [26], which is substantially lower than the ablation threshold for bulk $\mathrm{Au}$ $\left(\sim 0.6 \mathrm{~J} / \mathrm{cm}^{2}\right)[6]$. Here, we report a thermal-free ablation and modification for both metallic and dielectric nanostructures, utilizing the combination of a high-repetitionrate femtosecond oscillator and resonant plasmonic antennas. The nonthermal ablation mechanism also provides a means of laser-induced forward transfer [5,27] of materials with high resolution, enabling the fabrication of thin nanofilms with high surface quality.

\section{NEAR-FIELD ABLATION OF DIELECTRICS}

Let us first investigate plasmonic-antenna-enhanced ablation of fused silica $\left(\mathrm{SiO}_{2}\right)$. To this end, we manufacture Au bow-tie nanoantennas by focused-ion-beam milling of an Au film deposited on a sapphire substrate. A 3-nm-thick chromium (Cr) film is deposited first on the sapphire $\left(\mathrm{Al}_{2} \mathrm{O}_{3}\right)$, acting as an adhesion layer for the $\mathrm{Au}$ film. The bow tie consists of tip-to-tip oriented triangles with a length of $200 \mathrm{~nm}$, a height of $135 \mathrm{~nm}$, and an apex angle of $40^{\circ}$. After fabrication of the Au antenna, we deposited a 5-nm $\mathrm{SiO}_{2}$ layer to cover the Au structures. A Ti:sapphire oscillator with a repetition rate of $100 \mathrm{MHz}$, and central wavelength of $820 \mathrm{~nm}$ is then used for irradiating the nanostructures. The experiments are done in vacuum at a background pressure of $10^{-3}$ mbar. Additionally and to prevent oxide contamination, the vacuum chamber is purged with nonreactive pure gases such as argon and nitrogen prior to the experiments. The laser peak intensity $\sim 2.5 \times 10^{11} \mathrm{~W} / \mathrm{cm}^{2}$ is estimated from the average power, which is directly measured after the focus. Here, a pulse duration of $10 \mathrm{fs}$ is measured via the dispersion-scan technique [28], and a waist size of $6 \mu \mathrm{m}$ is retrieved from a $z$ scan of the surface third harmonic generation. The laser polarization is aligned parallel to the longitudinal axis of the nanoantennas. For the employed laser intensities, we feel confident in neglecting the optical breakdown of the dielectric layer [29].

Figures 1(a) and 1(b) show the scanning electron microscopy (SEM) pictures of a nanoantenna before and after $t_{i}=50 \mathrm{~min}$ of laser irradiation. The comparison reveals that a transparent ellipsoidal nanostructure is produced by laser illumination in the gap between the adjacent tips. To gain further insight into this laser-produced nanostructure, we analyze the top-view image taken with the SEM, i.e., in the $x-y$ plane shown in Fig. 2(a). Moreover, Fig. 2(b) depicts a cross-sectional view of the nanostructure topography in the $x-z$ plane. This view is made accessible by milling with a $\mathrm{Ga}^{+}$-based focused ion beam. One can see from both views [squared area in Figs. 2(a) and 2(b)] that the deposited nanostructure is not a hollow bubble. In fact, the entire gap region is filled by material deposit.

In order to discriminate the chemical elements of this laser-induced deposition, we further apply energydispersive $x$-ray spectroscopy (EDS). The EDS element
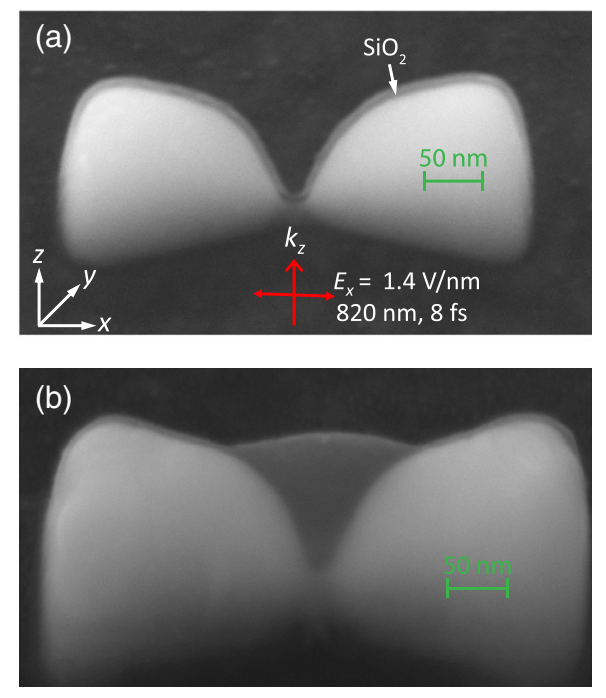

FIG. 1. SEM images of an Au nanoantenna with $\mathrm{SiO}_{2}$ dielectric coating in perspective view. (a) Pristine, (b) after 50 min of laser irradiation. 
(a)

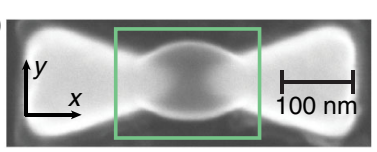

(c)

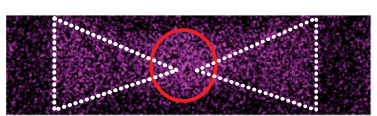

(e)

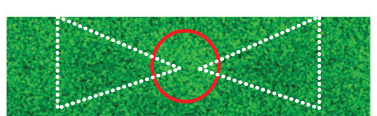

(g)

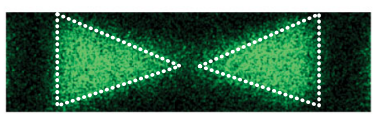

(i)

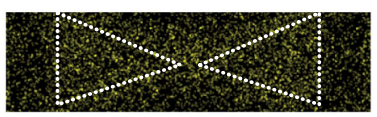

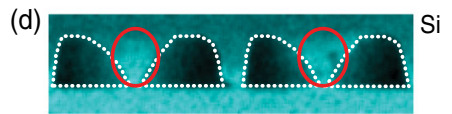
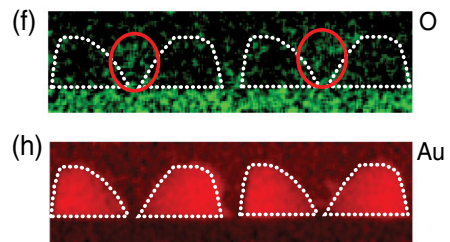

(j) $\because \cdots \cdots \cdot y^{\mathrm{Cr}}$

FIG. 2. SEM images and EDS maps. SEM images of bow-tie nanoantennas after $30 \mathrm{~min}$ of laser irradiation in the (a) $x-y$ plane and (b) the $x-z$ plane. (c)-(j) EDS element maps of the laser exposed nanostructure in the $x-y$ plane (left row) and in the $x-z$ plane (right row), which shows that the generated nanostructure in the gap contains indeed $\mathrm{Si}$ and $\mathrm{O}$, as indicated in the red circles in (c)-(f).

maps in the $x-y$ plane (left row) and the $x-z$ plane (right row) are shown in Figs. 2(c)-2(j). The dashed curves depict the outline of the nanoantennas. The analysis reveals that the chemical elements in the generated bubble-shaped nanostructure in the gap region are silicon [Figs. 2(c) and 2(d)] and, with lower visibility, oxygen [Figs. 2(e) and 2(f)]. As mentioned above, these two elements originate from the $\mathrm{SiO}_{2}$ coating. After laser illumination, in contrast, the $\mathrm{Au}$ content [Figs. 2(g) and 2(h)] remains confined to the original bow-tie shape. The complete absence of $\mathrm{Au}$ in the deposit proves that there is no ablation of the Au nanostructure itself. Finally, the distribution of $\mathrm{Cr}$ [Figs. 2(i) and 2(j)] clearly shows the 3-nm chromium adhesion layer.

The SEM micrographs in Figs. 1 and 2 further indicate that the ablation is localized at the apex of the nanostructure. This finding corroborates the absence of thermal effects. To this end, it is important to understand that plasmonic currents in the metallic skin layer and subsequent thermal diffusion are often considered the primary damage mechanism of nanoantennas [30]. In the presence of a $\mathrm{SiO}_{2}$ overcoating, one would then expect that the $\mathrm{Au}$ nanostructures need to be heated close to the boiling point (Au: $2973 \mathrm{~K}, \mathrm{SiO}_{2}: 3223 \mathrm{~K}$ ) to explain any material ablation. The practical absence of gold in the deposit as well as the lack of any deformation in the Au antennas therefore confirms that their temperature is still well below the boiling point of our hybrid system. By using the twotemperature model [31], the temperature of the Au nanoparticles is estimated to be approximately $500 \mathrm{~K}$, i.e., far below the onset of laser-induced melting or explosive boiling of $\mathrm{Au}$ [32].

(a)

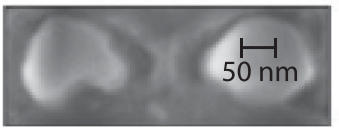

(c)

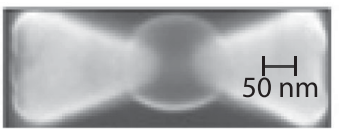

(b)

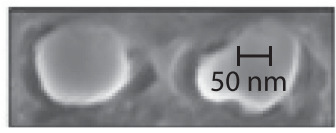

(d)

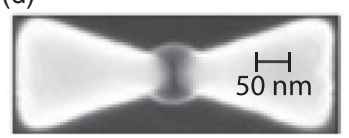

FIG. 3. SEM images of bow-tie nanoantennas with different heat capacities and with a $5-\mathrm{nm}$ coating after modification by $8 \mathrm{fs}$ (a),(c) and 16 fs (b),(d) laser pulses from an oscillator. The thicknesses of nanoantennas in (a),(b) has been reduced to $50 \mathrm{~nm}$ while the thickness in (c),(d) is $135 \mathrm{~nm}$. All the samples are illuminated by the same number of laser pulses.

\section{THERMAL MELTING VERSUS NEAR-FIELD ABLATION}

Usually, from strong laser illumination of gold nanoparticles, a structural modification is expected due to the transfer of energy from the heated electron gas into the lattice. This process typically leads to structural reshaping into a sphere, as imposed by the minimization of surface energy [33]. In order to evaluate this effect and concomitant electric near-field enhancement assisted local ablation, we reduce the bow-tie nanoantenna thickness from 135 to $50 \mathrm{~nm}$ as to reduce the heat capacity of the antenna structures [34]. While thermal energy diffusion is fast within the metallic nanoantenna heat sinking through the substrate acts as a bottleneck, given the 200 times lower thermal conductivity of sapphire. Figure 3(a) displays the SEM image of a 50-nm bow tie after irradiation by $10-\mathrm{fs}$ pulses in vacuum, which clearly indicates the near-spherical resulting surface that is expected to form after melting of the bow-tie antennas [35]. The Au antennas are clearly modified to nanospheres, while the remains of the dielectric coating still show a triangular footprint. Leaving the pulse energy constant while increasing the duration to 16 fs does not affect the melting of the low-heat-capacity antennas [Fig. 3(b)], whereas it noticeably reduces the ablation in the field-based interaction scenario [Fig. 3(d)]. Modification of the antenna thickness therefore allows switching between a thermal damage scenario and a nonlinear optical ablation mechanism that scales with local peak-field strength.

\section{COMPARISON BETWEEN $\mathrm{SiO}_{2}$-COATED AND UNCOATED Au ANTENNAS}

Previously, laser ablation mechanisms in dielectrics and metals had always been considered fundamentally different [36,37], with multiphoton absorption ruling the former and linear absorption dominating the latter. Here, we observe similar damage imposed by the same nonlinear mechanism for both dielectrics and metals. Figure 4(a) shows the SEM image of an uncoated Au bow tie after 50 min of illumination in vacuum. In principle, there appear to be several promising 
(a)

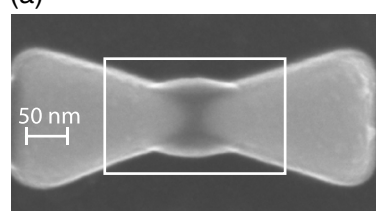

(c)

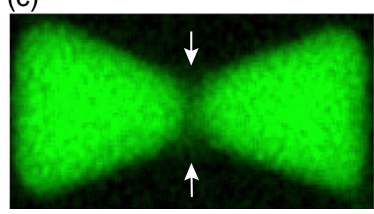

(b)

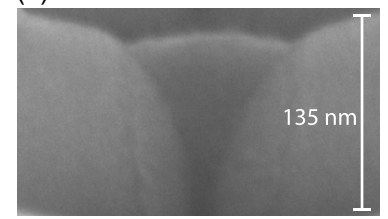

(d)

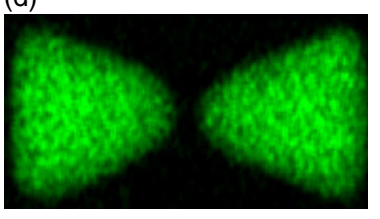

FIG. 4. Top-view (a) and enlarged side-view (b) SEM image of a pure Au nanoantenna after laser ablation. The thickness of the antenna is $135 \mathrm{~nm}$. EDS map showing the $\mathrm{Au}$ content of an ablated (c) and pristine (d) Au antenna.

ways to reduce or avoid the thermal damage observed in Figs. 3(a) and 3(b), e.g., the use of pulses with decreased duration or shorter wavelength as well as an increase of the heat capacitance of the antennas. Here, we choose the latter way and increase the thickness of the Au antennas from 50 to $135 \mathrm{~nm}$. One can observe qualitatively identical results when compared to the case of $\mathrm{a} \mathrm{SiO}_{2}$ coating, i.e., the ablation is confined to the sharpest tips, and no damage is visible in the shanks of the antenna. The squared area in Fig. 4(a) is shown enlarged in Fig. 4(b) to show the gap region at a tilt angle of $45^{\circ}$. Figures 4(c) and 4(d) show the two-dimensional EDS maps of the Au bow-tie nanoantennas. Compared to the pristine antenna [Fig. 4(d)], we can confirm that the deposit in the feed gap, as indicated by the white arrows in Fig. 4(c), is indeed Au. This kind of low-density metal deposit has been observed previously by nanosecond laser ablation [38]. The highly localized deformation yet again confirms that the ablation does not originate from thermal processes, as induced currents are expected to be maximal in the shank of the bow-tie nanoantennas rather than at the tips [39].

\section{VARIATION OF LINEAR EXTINCTION SPECTRA BY THERMAL MELTING AND NEAR-FIELD ABLATION}

In order to further analyze near-field-enhanced local ablation and the thermal melting mechanism, we also measure the extinction spectra of pure Au antennas before and after laser modification. Here, an ultrabroadband Ti: sapphire femtosecond oscillator $(650-1000 \mathrm{~nm})$ is utilized as a white light source to enable an in situ linear transmission measurement. The green squares in Fig. 5 show a typical plasmonic resonance of pristine nanoantennas centered at $830 \mathrm{~nm}$. As thermal melting completely reshapes the nanoantennas [inset, Fig. 5(a)], the complete vanishing of the plasmonic resonance results [blue circles, Fig. 5(a)]. In contrast, in the case of near-field enhanced ablation, reshaping is limited to the tip region, without modification of other parts of nanoantennas. This
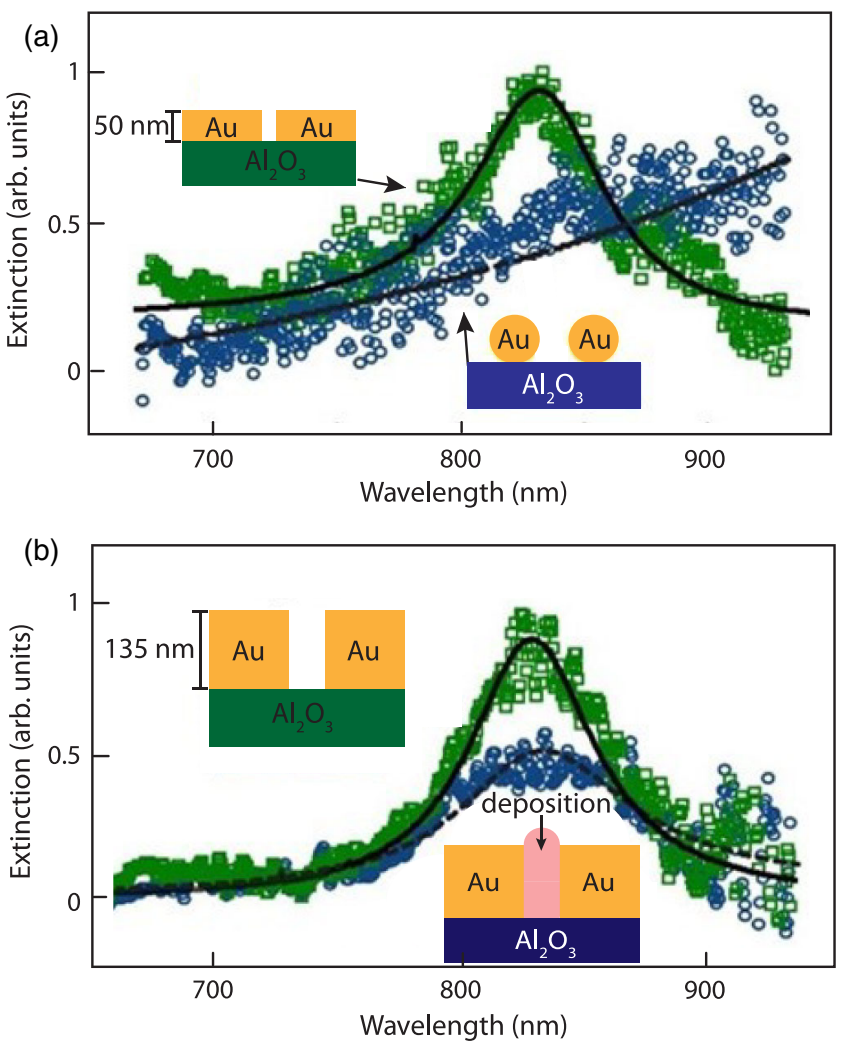

FIG. 5. (a) Experimentally measured extinction spectra of plasmon resonant $\mathrm{Au}$ nanoantennas before (green squares) and after (blue circles) laser irradiation. Thermal melting reshapes the bow-tie nanoantennas into nanospheres, and thus the plasmonic resonance disappears. (b) The increase of heat capacity induces the transition from thermal melting to near-field-enhanced nonthermal ablation, leading to the deposit of a low-density thin film in the feed gap. Extinction spectra of nanoantennas after near-field ablation (blue circles) still show the plasmonic resonance, yet attenuated with respect to the pristine antennas (green squares).

mechanism apparently weakens the plasmonic resonance, but does not completely remove it [blue circles, Fig. 5(b)]. This attenuation may be attributed to the Au deposit in the feed gap.

\section{THIRD-HARMONC GENERATION EFFICIENCY OF NANOANTENNAS}

An FDTD-based numerical simulation of the near-field distribution indicates that the peak intensity is comparable regardless of the presence of a $\mathrm{SiO}_{2}$ overcoating [Figs. 6(a) and 6(b)]. In order to verify the near-field enhancement, we measure the surface third-harmonic generation (THG) [40-42] as a function of laser electric field direction [Fig. 6(c)]. The THG counts are normalized to the signal at $\theta=90^{\circ}$. Here, the parameter $\theta$ is defined as the crossing angle between the laser polarization and the long axis of the bow tie ( $x$ axis). Independently of the presence of an overcoating, we observe that the THG shows an enhancement 

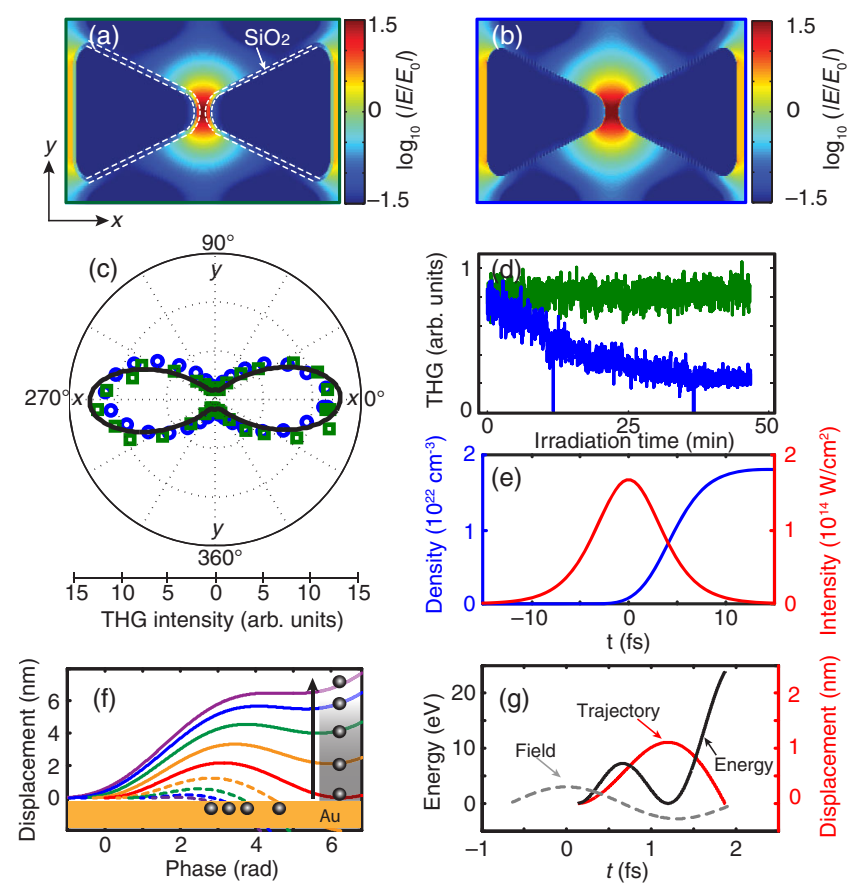

FIG. 6. Simulations of near-field distribution of the antenna with (a) and without (b) $\mathrm{SiO}_{2}$ coating. (c) Gap-plasmon-enhanced surface THG from $\mathrm{SiO}_{2}$-coated (green squares) and metallic (blue circles) antenna as a function of the laser polarized direction. (d) Temporal evolution of surface THG with long-term irradiation (green curve, coated antenna; blue curve, metallic antenna). (e) Electron density at the surface of fused silica (blue) and the laser intensity envelope (red). (f) Representative trajectories of electrons tunneling at different phases. Some electrons are sent back to the $\mathrm{Au}$ surface, while others remain in vacuum. The black arrow indicates the direction of electron velocity. The electron density also decreases along the black arrow, meaning that most of the quiver electrons are close to the surface after a single laser cycle. (g) Energy (black curve) and trajectory (red curve) of the most energetic returning electrons.

when the laser polarization is parallel to the bow-tie axis, i.e., $\theta=0^{\circ}$. Figure $6(\mathrm{~d})$ plots the temporal evolution of the THG flux for long-term laser irradiation in vacuum. The THG emission from pure Au nanoantennas (blue curve) is progressively decreasing with irradiation time. This behavior is attributed to the removal of $\mathrm{Au}$ atoms from the tips, resulting in an increase of the radius of curvature and gap size, which reduces the surface charge density, and thus the near-field enhancement factor. In contrast, the bow tie with $\mathrm{SiO}_{2}$ overcoating is capable of maintaining a high THG flux for nearly an hour (green curve). These findings appear to be in concord with the previous observations, i.e., the coated dielectric nanofilm can efficiently prevent the nearfield ablation of $\mathrm{Au}$ atoms.

The electric field enhancement is crucial for the whole ablation process, therefore, we also simulate the near-field distribution around pristine as well as ablated bow-tie antennas. After the near-field-enhanced ablation, the most significant changes to the nanoantenna include the increase
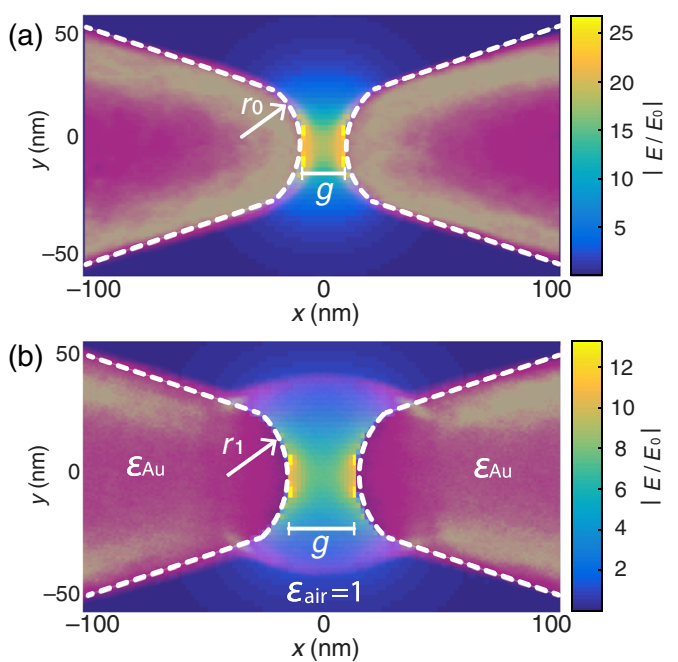

FIG. 7. Numerical simulations of the near-field enhancement $\left|E / E_{0}\right|$ in the gap region of a pristine (a) and an ablated (b) gold bow-tie nanoantenna. The geometrical parameters for simulations (dashed white curves) are in excellent agreement with the real size of the nanostructures (embedded false-color SEM images) before (a) and after (b) ablation. Note that $r_{0}$ and $r_{1}$ are the radius of curvature at the tips, $g$ is the gap size, $\varepsilon_{\text {Au }}$ and $\varepsilon_{\text {air }}$ denote the dielectric constant of gold bulk and air, respectively.

of radius of curvature $(r)$ of the tips, and the increase of gap size $(g)$. Our pristine bow-tie nanoantennas are fabricated to high precision with the following parameters: $r_{0}=20 \mathrm{~nm}$, $g=20 \mathrm{~nm}$; cf. background SEM image in Fig. 7(a). We estimate from a high-resolution SEM image [background image in Fig. 7(b)], that after ablation, the radius of curvature is increased to $r_{1} \sim 25 \mathrm{~nm}$, and thus the gap size is stretched to $g \sim 30 \mathrm{~nm}$. Figures 7(a) and 7(b) show the simulated field distribution in the gap region of a pristine and an ablated bow-tie nanoantenna, with the dielectric constant of the sapphire substrate set to be $\varepsilon_{\text {sapphire }}=3$. From Figs. 7(a) and 7(b), we can see that the field enhancement factor is evidently reduced by a factor of 2 due to the change of the bow-tie geometry.

\section{DISCUSSION OF NEAR-FIELD ABLATION MECHANISMS}

From the previous discussion of experimental results we can exclude plasmonic currents or global heating of the nanostructures as the dominant damage mechanisms, provided only that the antenna has a sufficient heat capacitance to accommodate the absorbed energy fraction of a single laser pulse. The results obtained with the overcoating also rule out field ion evaporation $[43,44]$ and ion electromigration [45] as possible reasons for the observed ablation processes.

In order to explain the observed antenna-assisted ablation, we consider several well-established mechanisms known from the ablation of bulk materials by femtosecond 
pulses. Conventional thermodynamics induced strong ablation at high fluence and long pulse duration ( $\geq$ few picoseconds) cannot sufficiently explain the femtosecond ablation in our experiments. Given the ultrashort pulse duration, laser fluence at the typical damage threshold of gold and silica amounts to peak intensities above $10^{13} \mathrm{~W} / \mathrm{cm}^{2}$. At this intensity level, nonlinear optical effects such as multiphoton-tunneling ionization and electron emission become significant for few-cycle pulses even in the presence of linear absorption. In fact, energy transfer time from the electrons to ions by collisions requires several picoseconds, which is much longer than the femtosecond pulse duration. Therefore, the femtosecond ablation at low fluence is rather attributed to material nonthermal unbonding and Coulomb-force-driven electrostatic ablation $[46,47]$, i.e., a nonthermal and rather gentle ablation process, with a much lower ablation rate than observed in a thermal regime. In this ablation scenario, the intense laser pulse excites a nonequilibrium plasma, characterized by the short-lived (about $1 \mathrm{ps}$ ) presence of hot electrons residing in a cold lattice. Although the ions in the lattice remain at room temperature, they experience a sudden change of potential energy and thus may be transiently unbound [46]. Under these transient conditions, a certain amount of hot electrons can escape from the surface in a second excitation step, which creates an electrostatic field between the ejected electrons and the remaining ionized atoms due to the charge separation. While this is certainly a rare process, the resultant electric field may occasionally pull individual ions out of the target [47], resulting in a slow and gentle removal of the first few monolayers (several nanometers).

As shown in Fig. 6(a), the field enhancement factor $\left|E / E_{0}\right|$ around the surface layer of the $\mathrm{SiO}_{2}$ apex reaches 25 , corresponding to a peak intensity of $I_{0}=1.5 \times 10^{14} \mathrm{~W} / \mathrm{cm}^{2}$. Theoretical predictions indicate that $\mathrm{SiO}_{2}$ will experience multiphoton absorption, tunneling ionization, and possibly subsequent avalanche processes in such strong electric fields. Using the Keldysh [48,49] formalism, we compute the adiabaticity parameter for solids $\gamma=\omega \sqrt{m E_{g}} / q_{e} E$. Using $m=0.635 m_{e}$ as the effective electron mass and $E_{g}=$ $8.9 \mathrm{eV}$ as the band gap of $\mathrm{SiO}_{2}, \gamma$ is then estimated as 0.45 , indicative of the prevalent role of tunneling ionization, and, subsequently, possible avalanche ionization. The temporal evolution of electron density $\rho(t)$ is given by [49]

$$
\frac{d \rho(t)}{d t}=\alpha \rho(t) \xi I(t)+\beta_{m}[\xi I(t)]^{m}-\frac{\rho(t)}{T} .
$$

For $\mathrm{SiO}_{2}, \alpha=8 \mathrm{~cm}^{2} / \mathrm{J}$ is the avalanche coefficient, $\beta_{m}=9.9 \times 10^{25} \mathrm{~cm}^{2 m-3} \mathrm{fs}^{m-1} / \mathrm{J}^{m}$ is the absorption coefficient with the photon number $m=6, \xi=0.66$ is the ratio of the maximum internal and the incident intensity, and $T=220 \mathrm{fs}$ is an electron effective relaxation time. The intensity envelope of the laser pulse is described as
$I(t)=I_{0} \operatorname{sech}^{2}[2 \ln (1+\sqrt{2}) t / \tau]$, with $\tau \sim 10$ fs denoting the pulse duration (full width at half maximum). By integrating Eq. (1), we find that the plasma density in fused silica [Fig. 6(e)] clearly exceeds the critical plasma density at $800 \mathrm{~nm} \rho_{\mathrm{cr}} \approx 10^{21} \mathrm{~cm}^{-3}$. As a result, the dielectric overcoating effectively becomes a metal-like overdense plasma.

In a second step, electrons can be ejected out of the surface due to Fowler-Nordheim tunneling [50-52]

$$
t(E)=\exp \left(-\frac{4}{3} \frac{\sqrt{2 q_{e} m_{e}}}{\hbar E} \phi^{3 / 2}\right) .
$$

Here, $\hbar$ is Planck's constant, and $\phi$ is the work function. For uncoated $\mathrm{Au}$ antennas, the work function $\phi_{\mathrm{Au}}=$ $4.83 \mathrm{eV}$. The work function of metallic silica is estimated by subtracting the band edge of fused silica from the ionization energy $I_{P}=13.6 \mathrm{eV}$, i.e., $\phi_{\mathrm{SiO}_{2}}=I_{p}-E_{g}=$ $3.7 \mathrm{eV}$ [53]. For a field strength of $30 \mathrm{~V} / \mathrm{nm}$, corresponding to an intensity of $1.5 \times 10^{14} \mathrm{~W} / \mathrm{cm}^{2}$, the tunneling probability $|t(E)|^{2}$ amounts to $\sim 1 \%$ and $\sim 3 \%$ for $\mathrm{Au}$ and metallic silica, respectively. From this calculation, we can see that, near the peak field strength, a large number of electrons $>10^{20} \mathrm{~cm}^{-3}$ can be ejected from the surface.

After tunneling out of the antennas, these free electrons are then accelerated by the laser field $E \times \cos (\omega t)$ of the remaining part of the pulse. The displacement of a free electron as a function of time is given by a semiclassical simple man's model [54],

$$
x(t)=\frac{q_{e} E}{m_{e} \omega^{2}}\left[-\cos (\omega t)-\omega\left(t-t_{0}\right) \sin \left(\omega t_{0}\right)+\cos \left(\omega t_{0}\right)\right] .
$$

Here, $\omega t_{0}$ is the phase at which the electron is released. Figure 6(f) shows the positions of electrons tunneling at different phases between $-\pi / 2$ and $\pi / 2$. We find that the electrons emitted after the peak field strength $(\pi / 2>$ $\left.\omega t_{0}>0\right)$ can return to the surface. Figure $6(\mathrm{~g})$ shows the trajectory (red curve) and the energy (black curve) for the mostly energetic recolliding electrons. In our experiments, the maximum kinetic energy of the returning electrons is calculated to be $E_{k} \sim 3.17 \cdot U_{p} \sim 23 \mathrm{eV}$ [Fig. 6(g), black curve], where $U_{p}=q_{e}^{2} E^{2} / 4 m_{e} \omega^{2}$ is the ponderomotive energy. Some part of the returned kinetic energy will be deposited into the surface, a process termed as vacuum heating of the plasma [55], which can additionally excite surface electrons. The high excitation of surface electrons by the intense light wave as well as the returning energetic electrons can rapidly change the potential energy of surface atoms and molecules, which might be transiently unbound on a subpicosecond time scale. This unbonding associated with electron excitation and ejection occurs only at the surface and will not change the shape as 
well as the size of nanoparticles. Such a surface unbonding is a significant feature of interaction between plasmonic nanoparticle and femtosecond laser, which recently has been extensively reported [56-58]. Some atoms and molecules with extreme initial thermal velocity in the highenergy tail of the Maxwell-Boltzmann distribution may escape from the surface within a subpicosecond time window, i.e., on a time scale shorter than the electron relaxation time.

The electrons liberated prior to the maximum field strength $\left(-\pi / 2<\omega \mathrm{t}_{0} \leq 0\right)$, as well as the secondary electrons will remain in the vacuum between the antenna tips, leaving an energetic electron train near the surface. As shown in Fig. 6(f), the electron density decreases along the black arrow, i.e., most of the free electrons are rather close to the surface after quiver oscillation. Therefore, an electrostatic field is produced between the escaped electrons and the positively charged nanotips [57-62], as schematically illustrated in Fig. 8. The induced electrostatic field may be strong enough to occasionally pull individual ions from the antenna structure. This process is the standard picture of the electrostatic ablation mechanism, which has been widely investigated [60-62]. The time for electrostatic ablation, i.e., the ion escape time, is given by $[47,62]$,

$$
t_{\mathrm{esc}}=\frac{2}{\omega_{\mathrm{pe}}} \sqrt{\frac{m_{i}}{m_{e}}} \sqrt{\frac{\varepsilon_{\mathrm{esc}}}{\left(\varepsilon_{\mathrm{esc}}-\varepsilon_{b}\right)}} .
$$

Here, $\omega_{\mathrm{pe}}=1.37 \times 10^{16} \mathrm{~Hz}$ is the electron plasma frequency of $\mathrm{Au}, m_{i}=3.25 \times 10^{-25} \mathrm{~kg}$ is the mass of an $\mathrm{Au}$ atom. The barrier potential of the $\mathrm{Au}$ ion is $\varepsilon_{b}=$ $8.2 \mathrm{eV}$ [63]. The electron-electron equilibration time is about $1 / \omega_{\mathrm{pe}} \sim 0.07 \mathrm{fs}$, much shorter than the pulse duration. Therefore, the electrons are in thermodynamic equilibrium during the laser pulse; and the surface plasmon-enhanced emission of hot electrons follows a Maxwellian distribution. A portion of these electrons at

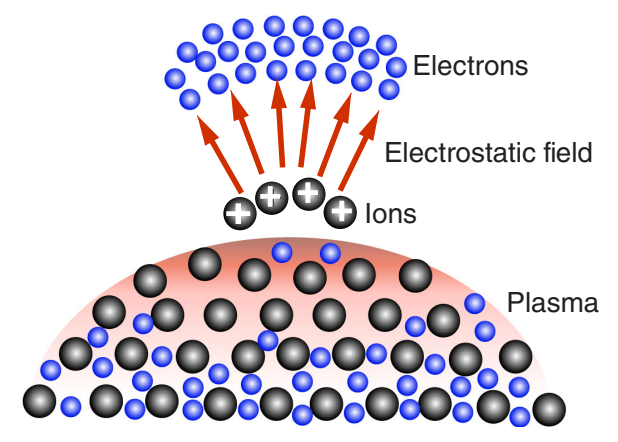

FIG. 8. Schematic illustration of the electrostatic ablation mechanism. The plasmonic near-field enhancement at the surface layer results in strong ionization and hot electron emission. After the pulse, the electrons remaining in the vacuum create an intense electrostatic field, which may then pull individual ions out of the surface. the high-energy tail of Maxwellian distribution exceeds the ion potential barrier and may be capable of pulling out the ions. If we assume an electron energy of $10 \mathrm{eV}$, the ablation time of the Au ion is estimated to be $t_{\mathrm{esc}} \sim 200 \mathrm{fs}$, which is evidently shorter than the electron-phonon coupling time. Similar considerations can be performed for $\mathrm{SiO}_{2}$, which is more susceptible to electrostatic ablation due to the much smaller atomic mass in the molecule.

In our experiments, the fluence at the tips is estimated to be $\sim 1 \mathrm{~J} / \mathrm{cm}^{2}$, which is exactly located in the regime of reported gentle ablation for both gold and silica [64]. Our main observations (Figs. 1-4) also agree with the proposed gentle ablation mechanisms. First, we propose that only the atoms or ions at the high-energy tail of Maxwellian distribution contribute to the ablation. This explains the rather low ablation rate, i.e., one needs several minutes to remove a few nanometers. Second, the decisive excitation mechanism occurs on a subpicosecond time scale, which is much shorter than the lattice heating time. This explains the absence of collateral thermal damage of the nanostructures. Third, the field electron emission and acceleration closely depend on the peak intensity, explaining the dependence of ablation on the pulse duration [Figs. 3(c) and 3(d)].

\section{CONCLUSION}

In summary, we introduce two important design improvements for resonant plasmonic nanoantennas, which both contribute to an extended lifetime of these structures. First, we show that a dielectric overcoating can prevent a degradation of the metallic antenna structure if melting or boiling of the antenna can be suitably prevented. We then show that the latter can be accomplished if the heat capacitance of the plasmonic system is chosen large enough to host the absorbed energy of an individual laser pulse without exceeding the melting temperature of gold. The latter can be achieved by manufacturing antennas of extended thickness. Such carefully designed antennas can be operated for nearly an hour without showing any sign of degradation in their nonlinear optical response.

Apart from the demonstrated stability of the performance of our antennas, the conducted experiments also provide insight into the rich interaction physics of plasmonic nanoantennas. While previously Ohmic losses of the resonantly induced currents were considered the major source of local heating, we explore an additional nonlinear optical damage mechanism that is confined to regions of maximum field enhancement. At the antenna tips, the field enhancement is so strong that near-unity Keldysh parameters can be reached, which enables tunneling of electrons out of the surface of the antennas. These free electrons may then experience a further acceleration by the oscillating electric field. It is interesting to see a class of highly nonlinear interaction processes at work at nanojoule pulse energy levels, whereas these processes have usually been investigated with large amplified laser systems. Most 
importantly, however, one can influence the relative strength of linear and nonlinear interaction processes as well as their impact on the lifetime of the antenna by suitable design. We therefore think that our findings present alternatives for applications of these highly interesting plasmonic structures. Another interesting aspect is the suggested very cold nature of the ablated gold atoms. Cold ablation processes may therefore enable the construction of atom or ion sources with extremely narrow energy distribution.

\section{ACKNOWLEDGMENTS}

We are thankful for the funding support from the Deutsche Forschungsgemeinschaft (DFG) under Grant No. KO 3798/4-1, from the Centre for Quantum Engineering and Space-Time Research (QUEST), from Lower Saxony through "Quanten-und Nanometrologie" (QUANOMET, project Nanophotonik) from the National Research Foundation of the Republic of Korea (NRF2012R1A3A1050386), from the European Union through the VOXEL FET Open, from the French Ministry of Research through the ANR grants "NanoImagine", "IPEX", "HELLIX", PACHA and from the C'NANO research program through the NanoscopiX grant, and the LABEX "PALM" through the grants "Plasmon- $X$ " and "HILAC". We acknowledge the financial support from the French ASTRE program through the "NanoLight" grant and the support from the DGA RAPID program through the "SWIM LASER" grant. L.S. is grateful to Professor Jeremy Baumberg from the University of Cambridge for stimulating discussion.

[1] D. Perez and L. J. Lewis, Ablation of Solids Under Femtosecond Laser Pulses, Phys. Rev. Lett. 89, 255504 (2002).

[2] C. Kerse et al., Ablation-cooled material removal with ultrafast bursts of pulses, Nature (London) 537, 84 (2016).

[3] F. Bauer, A. Michalowski, T. Kiedrowski, and S. Nolte, Heat accumulation in ultra-short pulsed scanning laser ablation of metals, Opt. Express 23, 1035 (2015).

[4] F. Takahashi, K. Miyamoto, H. Hidai, K. Yamane, R. Morita, and T. Omatsu, Picosecond optical vortex pulse illumination forms a monocrystalline silicon needle, Sci. Rep. 6, 21738 (2016).

[5] D. A. Willis and V. Grosu, Microdroplet deposition by laserinduced forward transfer, Appl. Phys. Lett. 86, 244103 (2005)

[6] B. C. Stuart, M. D. Feit, S. Herman, A. M. Rubenchik, B. W. Shore, and M. D. Perry, Optical ablation by high-power short-pulse lasers, J. Opt. Soc. Am. B 13, 459 (1996).

[7] M. D. Perry, B. C. Stuart, P. S. Banks, M. D. Feit, V. Yanovsky, and A. M. Rubenchik, Ultrashort-pulse laser machining of dielectric materials, J. Appl. Phys. 85, 6803 (1999).

[8] P. Mulvaney, Nanoscience vs nanotechnology-Defining the field, ACS Nano 9, 2215 (2015).
[9] Y. Luan, V. Zorba, X. Mao, R. Zheng, and R. E. Russo, UV fs-ns double-pulse laser induced breakdown spectroscopy for high spatial resolution chemical analysis, J. Anal. At. Spectrom. 28, 743 (2013).

[10] C. Jabbour, J.-L. Lacour, M. Tabarant, A. Semerok, and F. Chartier, Development of a tip enhanced near-field laser ablation system for the sub-micrometric analysis of solid samples, J. Anal. At. Spectrom. 31, 1534 (2016).

[11] M. V. Zoriy and J.S. Becker, Near-field laser ablation inductively coupled plasma mass spectrometry: A novel elemental analytical technique at the nanometer scale, Rapid Commun. Mass Spectrom. 23, 23 (2009).

[12] A. A. Milner, K. Zhang, and Y. Prior, Floating tip nanolithography, Nano Lett. 8, 2017 (2008).

[13] S. Ghorai, C. A. Seneviratne, and K. K. Murray, Tipenhanced laser ablation sample transfer for biomolecule mass spectrometry, J. Am. Soc. Mass Spectrom. 26, 63 (2015).

[14] P. Muhlschlegel, H. J. Eisler, O. J. F. Martin, B. Hecht, and D. W. Pohl, Resonant optical antennas, Science 308, 1607 (2005).

[15] L. Novotny and N. Van Hulst, Antennas for light, Nat. Photonics 5, 83 (2011).

[16] T. H. Taminiau, R. J. Moerland, F. B. Segerink, L. Kuipers, and N. F. Van Hulst $\lambda / 4$ resonance of an optical monopole antenna probed by single molecule fluorescence, Nano Lett. 7, 28 (2007).

[17] M. W. Knight, H. Sobhani, P. Nordlander, and N. J. Halas, Photodetection with active optical antennas, Science 332, 702 (2011).

[18] M. L. Brongersma, Y. Cui, and S. Fan, Light management for photovoltaics using high-index nanostructures, Nat. Mater. 13, 451 (2014).

[19] J. A. Schuller, T. Taubner, and M. L. Brongersma, Optical antenna thermal emitters, Nat. Photonics 3, 658 (2009).

[20] J. Y. Suh, M. D. Huntington, C. H. Kim, W. Zhou, M. R. Wasielewski, and T. W. Odom, Extraordinary nonlinear absorption in 3D bowtie nanoantennas, Nano Lett. 12, 269 (2012).

[21] M. Sivis, M. Duwe, B. Abel, and C. Ropers, Extremeultraviolet light generation in plasmonic nanostructures, Nat. Phys. 9, 304 (2013).

[22] A. Kinkhabwala, Z. F. Yu, S. H. Fan, Y. Avlasevich, K. Mullen, and W. E. Moerner, Large single-molecule fluorescence enhancements produced by a bowtie nanoantenna, Nat. Photonics 3, 654 (2009).

[23] S. Kim, J. H. Jin, Y. J. Kim, I. Y. Park, Y. Kim, and S. W. Kim, High-harmonic generation by resonant plasmon field enhancement, Nature (London) 453, 757 (2008).

[24] H. Aouani, M. Rahmani, M. Navarro-Cía, and S. A. Maier, Third-harmonic-upconversion enhancement from a single semiconductor nanoparticle coupled to a plasmonic antenna, Nat. Nanotechnol. 9, 290 (2014).

[25] M. L. Juan, M. Righini, and R. Quidant, Plasmon nanooptical tweezers, Nat. Photonics 5, 349 (2011).

[26] C. Kern, M. Zürch, and C. Spielmann, Limitations of extreme nonlinear ultrafast nanophotonics, Nanophotonics 4, 303 (2015).

[27] P. Delaporte and A.-P. Alloncle, Laser-induced forward transfer: A high resolution additive manufacturing technology, Opt. Laser Technol. 78, 33 (2016). 
[28] M. Miranda, T. Fordell, C. Arnold, A. L'Huillier, and H. Crespo, Simultaneous compression and characterization of ultrashort laser pulses using chirped mirrors and glass wedges, Opt. Express 20, 688 (2012).

[29] S. Zherebtsov et al. Controlled near-field enhanced electron acceleration from dielectric nanospheres with intense fewcycle laser fields, Nat. Phys. 7, 656 (2011).

[30] B. Chimier, O. Uteza, N. Sanner, M. Sentis, T. Itina, P. Lassonde, F. Legare, F. Vidal, and J. C. Kieffer, Damage and ablation thresholds of fused-silica in femtosecond regime, Phys. Rev. B 84, 094104 (2011).

[31] O. Ekici, R. K. Harrison, N. J. Durr, D. S. Eversole, M. Lee, and A. Ben-Yakar, Thermal analysis of gold nanorods heated with femtosecond laser pulses, J. Phys. D 41, 185501 (2008).

[32] A. Plech, V. Kotaidis, M. Lorenc, and J. Boneberg, Femtosecond laser near-field ablation from gold nanoparticles, Nat. Phys. 2, 44 (2006).

[33] J. Wang, Y. T. Chen, X. Chen, J. M. Hao, M. Yan, and M. Qiu, Photothermal reshaping of gold nanoparticles in a plasmonic absorber, Opt. Express 19, 14726 (2011).

[34] S. Link and M. A. EI-Sayed, Shape and size dependence of radiative, non-radiative and photothermal properties of gold nanocrystals, Int. Rev. Phys. Chem. 19, 409 (2000).

[35] W. Albrecht, T.-S. Deng, B. Goris, M. A. van Huis, Sara Bals, and A. van Blaaderen, Single particle deformation and analysis of silica-coated gold nanorods before and after femtosecond laser pulse excitation, Nano Lett. 16, 1818 (2016).

[36] R. Stoian, A. Rosenfeld, D. Ashkenasi, I. V. Hertel, N. M. Bulgakova, and E. E. B. Campbell, Surface Charging and Impulsive Ion Ejection During Ultrashort Pulsed Laser Ablation, Phys. Rev. Lett. 88, 097603 (2002).

[37] K. Sokolowski-Tinten, J. Bialkowski, A. Cavalleri, D. von der Linde, A. Oparin, J. Meyer-ter-Vehn, and S. I. Anisimov, Transient States of Matter During Short Pulse Laser Ablation, Phys. Rev. Lett. 81, 224 (1998).

[38] K. Seal, M. A. Nelson, Z. C. Ying, D. A. Genov, A. K. Sarychev, and V. M. Shalaev, Growth, morphology, and optical and electrical properties of semicontinuous metallic films, Phys. Rev. B 67, 035318 (2003).

[39] G. Baffou, C. Girard, and R. Quidant, Mapping Heat Origin in Plasmonic Structures, Phys. Rev. Lett. 104, 136805 (2010).

[40] G. Vampa, B. G. Ghamsari, S. S. Mousavi, T. J. Hammond, A. Olivieri, E. Lisicka-Skrek, A. Yu Naumov, D. M. Villeneuve, A. Staudte, P. Berini, and P. B. Corkum, Plasmon-enhanced high-harmonic generation from silicon, Nat. Phys. 13, 659 (2017).

[41] L. P. Shi, B. Iwan, R. Nicolas, Q. Ripault, J. R. C. Andrade, S. Han, H. Kim, W. Boutu, D. Franz, T. Heidenblut, C. Reinhardt, B. Bastiaens, T. Nagy, I. Babushkin, U. Morgner, S.-W. Kim, G. Steinmeyer, H. Merdji, and M. Kovačev, Self-optimization of plasmonic nanoantennas in strong femtosecond fields, Optica 4, 1038 (2017).

[42] L. P. Shi, J. R. C. Andrade, H. Kim, S. Han, R. Nicolas, D. Franz, W. Boutu, T. Heidenblut, F. B. Segerink, B. Bastiaens, H. Merdji, S.-W. Kim, U. Morgner, and M. Kovačev, Investigating the origin of third harmonic generation from diabolo optical antennas, Appl. Phys. Lett. 111, 173102 (2017).
[43] H. J. Mamin, P. H. Guethner, and D. Rugar, Atomic Emission from a Gold Scanning-Tunneling-Microscope Tip, Phys. Rev. Lett. 65, 2418 (1990).

[44] C. S. Chang, W. B. Su, and T. T. Tsong, Field Evaporation between a Gold Tip and a Gold Surface in the Scanning Tunneling Microscope Configuration, Phys. Rev. Lett. 72, 574 (1994).

[45] H. Park, A. K. L. Lim, A. P. Alivisatos, J. Park, and P. L. McEuen, Fabrication of metallic electrodes with nanometer separation by electromigration, Appl. Phys. Lett. 75, 301 (1999).

[46] B. Rethfeld, D. S. Ivanov, M. E. Garcia, and S. I. Anisimov, Modelling ultrafast laser ablation, J. Phys. D 50, 193001 (2017).

[47] E. G. Gamaly, The physics of ultra-short laser interaction with solids at non-relativistic intensities, Phys. Rep. 508, 91 (2011).

[48] L. V. Keldysh, Ionization in the field of a strong electromagnetic wave, Sov. Phys. JETP 20, 1307 (1965).

[49] M. Mero, J. Liu, W. Rudolph, D. Ristau, and K. Starke, Scaling laws of femtosecond laser pulse induced breakdown in oxide films, Phys. Rev. B 71, 115109 (2005).

[50] Vu T. Binh, N. Garcia, and S. T. Purcell, Electron field emission from atom-sources: Fabrication, properties, and applications of nanotips, Adv. Imaging Electron Phys. 95, 63 (1996).

[51] P. M. Nagel, J. S. Robinson, B. D. Harteneck, T. Pfeifer, M. J. Abel, J. S. Prell, D. M. Neumark, R. A. Kaindl, and S. R. Leone, Surface plasmon assisted electron acceleration in photoemission from gold nanopillars, Chem. Phys. 414, 106 (2013).

[52] D. J. Park, B. Piglosiewicz, S. Schmidt, H. Kollmann, M. Mascheck, and C. Lienau, Strong Field Acceleration and Steering of Ultrafast Electron Pulses from a Sharp Metallic Nanotip, Phys. Rev. Lett. 109, 244803 (2012).

[53] O. Kostko, M. Ahmed, and Ricardo B. Metz, Vacuumultraviolet photoionization measurement and $a b$ initio calculation of the ionization energy of gas-phase $\mathrm{SiO}_{2}$, J. Phys. Chem. A 113, 1225 (2009).

[54] P. Dombi, A. Hörl, P. Rácz, I. Márton, A. Trügler, J. R. Krenn, and U. Hohenester, Ultrafast strong-field photoemission from plasmonic nanoparticles, Nano Lett. 13, 674 (2013).

[55] L. M. Chen, J. Zhang, Q. L. Dong, H. Teng, T. J. Liang, L.Z. Zhao, and Z. Y. Wei, Hot electron generation via vacuum heating process in femtosecond laser-solid interactions, Phys. Plasmas 8, 2925 (2001).

[56] A. Hu, P. Peng, H. Alarifi, X. Y. Zhang, J. Y. Guo, and W. W. Duley, Femtosecond laser welded nanostructures and plasmonic devices, J. Laser Appl. 24, 042001 (2012).

[57] A. Hu, Y. Zhou, and W. W. Duley, Femtosecond laserinduced nanowelding: Fundamentals and applications, Open Surf. Sci. J. 3, 42 (2011).

[58] L. O. Herrmann, V. K. Valev, C. Tserkezis, J. S. Barnard, S. Kasera, O. A. Scherman, J. Aizpurua, and J. Baumberg, Threading plasmonic nanoparticle strings with light, Nat. Commun. 5, 4568 (2014).

[59] S. Tao and $\mathrm{B}$. Wu, The effect of emitted electrons during femtosecond laser-metal interactions: A physical explanation for Coulomb explosion in metals, Appl. Surf. Sci. 298, 90 (2014). 
[60] S. Li, S. Li, F. Zhang, D. Tian, H. Li, D. Liu, Y. Jiang, A. Chen, and M. Jin, Possible evidence of Coulomb explosion in the femtosecond laser ablation of metal at low laser fluence, Appl. Surf. Sci. 355, 681 (2015).

[61] Laser-Induced Breakdown Spectroscopy, edited by S. Musazzi and U. Perini (Springer, New York, 2014).

[62] E. G. Gamaly, A. V. Rode, B. Luther-Davies, and V. T. Tikhonchuk, Ablation of solids by femtosecond lasers: Ablation mechanism and ablation thresholds for metals and dielectrics, electrostatic mechanism of ablation by femtosecond lasers, Phys. Plasmas 9, 949 (2002).

[63] H. J. Mamin, P. H. Guethner, and D. Rugar, Atomic Emission from a Gold Scanning-Tunneling-Microscope Tip, Phys. Rev. Lett. 65, 2418 (1990).

[64] M. E. Shaheen, J. E. Gagnon, and B. J. Fryer, Femtosecond laser ablation behavior of gold, crystalline silicon, and fused silica: A comparative study, Laser Phys. 24, 106102 (2014). 